\title{
A DIOS POR LA CIENCIA. TEOLOGÍA NATURAL EN EL FRANQUISMO
}

\section{Francisco Blázquez Paniagua}

IES Pedro de Valdivia, Villanueva de la Serena (Badajoz)

\section{RESUMEN}

En España, durante la dictadura franquista (1939-1975), la enseñanza y la divulgación de la ciencia estuvieron supeditadas a la religión católica y numerosas obras defendieron una visión teísta y creacionista de la biología que aceptaba el relato literal del Génesis y rechazaba la teoría de la evolución, especialmente en el problema del origen del ser humano. Este artículo aborda las principales obras y características de esta forma de pensamiento que reprodujo argumentos y metáforas propios de la teología natural predarwiniana, según la cual la Naturaleza estaba gobernada por Dios y los seres vivos eran producto de su diseño.

PALABRAS CLAVE: Teología natural. Biología teísta. Creacionismo. Creacionismo en España.

\section{TO GOD THROUGH SCIENCE. NATURAL THEOLOGY IN FRANCOISM}

\begin{abstract}
In Spain, during Franco's dictatorship (1939-1975) the teaching and divulgation of science were subordinated to the Catholic religion and many books defended a theistic and creationistic point of view of Biology that accepted a literal interpretation of the Book of Genesis and denied the theory of evolution, especially as it relates to human origin. This article is devoted to the main books and characteristics of this way of thinking which reproduced arguments and metaphors of the Pre-Darwinian Natural Theology, arguing that Nature was ruled by God and living organisms were the results of his design.
\end{abstract}

KEY WORDS: Natural Theology. Theistic Biology. Creationism. Creationism in Spain.

\section{TEOLOGÍA NATURAL Y LA BIOLOGÍA PREDARWINIANA}

La historia natural entre los siglos XVI y XIX estuvo presidida por la llamada teología natural, una forma de pensamiento que situaba a Dios tras el origen, la complejidad y las adaptaciones de los seres vivos. Fue especialmente importante en Inglaterra a finales del siglo XVIII y durante la primera mi- 
tad del siglo XIX, destacando entre sus obras más representativas: The Wisdom of God Manifested in the Works of the Creation (1691) de John Ray (1627-1705), Natural Theology, or Evidences of the Existence and Attributes of the Deity collected from the Appearances of Nature (1802) de William Paley (1743-1805) y Bridgewater Treatises, una serie de tratados de varios autores que se publicaron entre 1830 y $1840^{1}$. Eran notables obras de historia natural que describían desde el orden astronómico hasta la complejidad anatómica de los organismos, incluidos los humanos, y todo ello se mostraba como prueba de la existencia de un Creador con una sabiduría infinita que había diseñado minuciosamente cada criatura para vivir perfectamente adaptada a su entorno.

Aquella visión era coherente con el relato bíblico de la Creación al principio de los tiempos y con los pensamientos platónico y el aristotélico, según los cuales las especies eran entidades ideales e inmutables que formaban parte de una Naturaleza jerarquizada en complejidad y perfección conocida como Scala Naturae o Gran Cadena del Ser.

La teoría de la evolución por selección natural formulada por Alfred R. Wallace (1823-1913) y Charles Darwin (1809-1882) en 1858, y especialmente por este último en El origen de las especies (1859), mostró sólidas pruebas $\mathrm{y}$ argumentos a favor de que tanto el origen como las adaptaciones de los seres vivos eran el resultado de un proceso natural de selección biológica, lo que rompió con la idea de creación sobrenatural, diseño divino, esencialismo y orden jerárquico de la Naturaleza².

Tras un período de eclipse e incertidumbre que se extendió entre finales del siglo XIX y el primer tercio del siglo $\mathrm{XX}^{3}$, la teoría de la evolución por selección natural quedó plenamente aceptada por la mayor parte de las disciplinas biológicas durante las décadas de 1930 y 1940, en la síntesis evolucionista o teoría sintética de la evolución. A pesar de esto, diversas formas de pensamiento, en contextos diferentes pero dominados por creencias religiosas, han compartido los argumentos básicos de la teología natural predarwiniana, cuestio-

1 Una descripción de las argumentaciones de estas obras pueden encontrarse en AYALA, F. J. (2007), Darwin y el Diseño Inteligente. Creacionismo, Cristianismo y Evolución, Madrid, Alianza Editorial, pp. 31-41. Véase también: MAYR, E. (1982), The Growth of Biological Thought, Cambridge-Londres, Harvard University Press, pp. 103-105; y BowLER, P. J. (1989), Evolution. The History of an Idea, Berkeley-Londres, pp. 52-55.

2 Véase MAYr, E. (1992), Una larga controversia, Darwin y el darwinismo, Barcelona, Crítica, pp. 34-36 y 51-52.

3 Bowler, P. J. (1985), El eclipse del darwinismo, Barcelona, Labor. 
nando tanto la evolución como el proceso de selección natural, entre ellos, las oleadas de creacionismo en Estados Unidos o el nacionalcatolicismo de la dictadura franquista que surgió tras la guerra civil española (1936-1939).

\section{HEREJÍAS CIENTÍFICAS EN LA POSGUERRA}

En España, el evolucionismo en su versión darwinista se consideró el núcleo de la llamada biología «materialista y atea». Desde su tardía recepción en los años del Sexenio Revolucionario (1868-1874) hasta la Guerra Civil (1936-1939) la cuestión estuvo fuertemente ideologizada, ausente del debate científico y generó intensas polémicas, especialmente en el siglo XIX ${ }^{4}$.

Aunque algunos naturalistas y clérigos no vieron problema en compatibilizar evolución y religión católica durante el primer tercio del siglo $\mathrm{XX}^{5}$, el discurso dominante desde sectores eclesiásticos fue muy distinto debido a la preocupación por el éxito del «criterio materialista-evolucionista» entre estudiantes, cátedras y obras de ciencias naturales ${ }^{6}$. Como destacara el sacerdote y biólogo, Jaime Pujiula (1869-1958), las ideas materialistas, monistas y evolucionistas habían estado «envenenando» las ciencias naturales y la biología, desviando la ciencia «de su verdadero ideal que es ascender por el conocimiento de las criaturas al conocimiento de su Creador» ${ }^{7}$.

El desenlace de la Guerra Civil propició el mejor escenario para reconducir esta situación y hacer realidad el sueño del padre Pujiula. En la posguerra la defensa del evolucionismo fue un elemento más de aversión y censura

4 Véanse, por ejemplo, algunos de los primeros trabajos sobre el darwinismo en España: NúÑEZ, D. (1977), El darwinismo en España, Madrid, Castalia; y GLICK, T. F. (1982), Darwin en España, Barcelona, Península. Una historiografía crítica completa se encuentra en Catalá, J. (2009), Cuatro décadas de historiografía del evolucionismo en España, Asclepio. Revista de Historia de la Medicina y de la Ciencia, 61, (2), pp. 9-66.

5 BlÁZQueZ, F. (2007), Notas sobre el debate evolucionista en España (1900-1936), Revista de Hispanismo Filosófico, 12, pp. 40-41.

6 Sobre la cruzada antievolucionista en las décadas anteriores a la Guerra Civil véase PElayo, F. (2002), Darwinismo y antidarwinismo en España (1900-1939): la extensión y crítica de las ideas evolucionistas. En PUIG-SAMPER, RUIZ, R. y GALERA, A. (eds.), Evolucionismo y Cultura. Darwinismo en Europa e Iberoamérica, Editora Regional de Extremadura/UNAM/Doce Calles, pp. 267-283; y BLÁZQUEZ (2007), pp. 23-44.

7 Pujiula, J. (1949), Manual Completo de Biología Moderna Macro y Microscópica, Barcelona, Tip. Cat. Casals, p. 5. Esta edición reproduce los prólogos de ediciones anteriores, la cita pertenece al de la primera edición de 1927. 
hacia personas e instituciones ligadas al periodo republicano, así por ejemplo, en un alegato contra la Institución Libre de Enseñanza, el Museo de Ciencias Naturales era considerado un "vivero de "transformistas" materialistas, que han arrancado en libros y cátedras tantas creencias con fáciles declamaciones contra el Génesis, a base de atractivas novelas darwinistas» ${ }^{8}$.

Las opiniones y directrices políticas sobre el evolucionismo y la concepción materialista de la biología fueron muy explícitas durante y tras la Guerra Civil. El ministro de educación del primer gobierno de Franco, Pedro Sainz Rodríguez (1897-1986), se refirió en 1938 a la teoría de la evolución como una «estrella apagada $\rangle^{9}$, y el segundo, José Ibáñez Martín (1896-1969), en el memorable discurso de inauguración del CSIC (1940), proclamó una ciencia católica y liquidó las «herejías científicas» afirmando que «los errores no pueden constituir ciencia, ni existe para ellos libertad científica» ${ }^{10}$. Estos errores habían estado señalándose durante décadas en las obras religiosas y se encontraban como había indicado Pujiula - en las ciencias naturales y la biología pues en estos campos «se cruzan las espadas de diversos contendientes» ${ }^{11}$.

\section{BiOLOGÍA TEÍSTA EN LAS AULAS}

La educación fue uno de los pilares de adoctrinamiento en la «nueva España» y la enseñanza media tenía especial importancia dado que sus contenidos profundizaban en aspectos claves que chocaban de lleno con la ortodoxia católica. Las consignas de Ibáñez Martín se materializaron plenamente en algunos manuales de ciencias naturales que incluían oraciones y pasajes del Génesis, mientras los catecismos argumentaban contra el evolucionismo, el darwinismo o la ciencia materialista ${ }^{12}$.

8 MARtín-SÁnchez, F. (1940), Origen, ideas e historia de la Institución Libre de Enseñanza. En AAVV (1940), Una poderosa fuerza secreta. La Institución Libre de Enseñanza, San Sebastián, Editorial Española, pp. 119-120. Consultado el 12/04/2010 en http://www. filosofia.org/aut/ile/1940p031.htm\#a15.

9 Discurso en la Universidad de Santiago, junio de 1938, citado en AlTED, A. (1984), Política del nuevo estado sobre el patrimonio cultural y la educación durante la Guerra Civil española, Madrid, Dirección General de Bellas Artes y Archivos del Centro Nacional de Información Artística, Arqueológica y Etnológica, p. 61.

10 Memoria de la Secretaría General 1940-1941, CSIC, Madrid, 1942, p. 32.

11 Pujiula (1949), p. 5. Véase nota 7.

12 Véase, por ejemplo, Nuevo Ripalda en la Nueva España (1951), reproducido por MIRET, E. y SÁdABA, J. (1998), El catecismo de nuestros padres, Plaza y Janés, p. 214. Los 
Aunque muchos textos religiosos proclamaron la armonía perfecta entre ciencia y fe, en realidad se trataba de una supeditación pues, como se reconocía, la fe era «la antorcha que guía a la humanidad por los campos de la investigación, mostrándole [a la ciencia] en muchos casos los escollos en que se puede naufragar» ${ }^{13}$. Estos escollos eran, entre otros, el evolucionismo (considerado un proceso general que implicaba a todo el universo), y el «transformismo» o «darvinismo» (restringido a los seres vivos y al ser humano) y tildado de «falso y herético» ${ }^{14}$.

En lo que respecta a los textos de ciencias naturales el hecho más relevante fue la supresión del tema de la evolución, presente en los cuestionarios (programas) de bachillerato anteriores a la guerra ${ }^{15}$. En algún caso quedaron frases con sentido evolucionista herederas de ediciones anteriores al final de la guerra, por ejemplo, en Curso de Historia Natural (1951) del catedrático de Fisiología Animal, Salustio Alvarado (1897-1981), se indicaba que las plantas y los mamíferos placentarios del Terciario evolucionaron o se diferenciaron para dar lugar a los representantes actuales. Esto no ocurrió con las afirmaciones relativas al origen humano, donde la neutra expresión «aparece el hombre» de ediciones anteriores a la guerra se sustituyó por «fue creado el hombre ${ }^{16}$. A este hecho hizo referencia Emilio Palafox (n. 1925), investigador del CSIC y sacerdote del Opus Dei, partidario de un evolucionismo finalista, cuando solicitó una reflexión a las autoridades educativas sobre las consecuencias negativas de esta censura para los alumnos universitarios ${ }^{17}$.

En general, las pocas referencias que se hicieron a la evolución en los textos de ciencias naturales de enseñanza media en la posguerra fueron para tildarla de doctrina o hipótesis: «el examen de dicha doctrina resulta que, lejos

párrafos contra el darwinismo también se encuentran en las ediciones de este catecismo anteriores a la Guerra Civil.

13 Villapún, M. (1949), El dogma católico. Quinto curso de Bachillerato, Madrid, Talleres gráficos Montaña, p. 16. Las ediciones de esta obra para $6 .^{\circ}$ de bachillerato (Plan del 57) continuaron hasta finales de los sesenta.

14 Villapún, M. (1964), El dogma católico. Sexto de Bachillerato, Madrid, Canales, p. 75. El término 'herético' no aparece en todas las ediciones anteriores.

15 Véanse los cuestionarios de Biología y Ciencias Naturales publicados en la Gaceta de Madrid, n. ${ }^{\circ}$ 252, 9 septiembre de 1935, pp. 1982-1983.

16 Alvarado, S. (1937 2a. ed.), Historia Natural para la Segunda Enseñanza en España e Hispanoamérica, Barcelona, pp. 335 y 338-339; AlvarAdO, S. (1951), Curso de Historia Natural para el Séptimo curso de Bachillerato (Plan 1938), Ed. Alvarado, pp. 112 y 119.

17 Palafox, E. (1953), Hacia una síntesis evolucionista, Theoría, 3-4, p. 122. Comunicación personal del autor 2/9/2003. 
de ser un sistema científico bien fundado en la experiencia, se reduce a un conjunto de hipótesis»» ${ }^{18}$.

Algunos manuales de ciencias naturales fueron en realidad una extensión del pensamiento religioso expresado en los catecismos. Entre ellos destacamos los del jesuita Vicente Muedra ${ }^{19}$, cuyo Ciencias Naturales. Segundo curso de Bachillerato (1956) mostraba una completa intercalación de pensamiento religioso en un libro de ciencias naturales ${ }^{20}$. La obra — que obtuvo mención honorífica del Ministerio de Educación Nacional - se iniciaba con una oración y un prólogo que señalaba su principal objetivo: introducir a los estudiantes en las ciencias para «llevarles por ellas a Dios». En la introducción se reproducía el primer capítulo del Génesis y una ilustración mostraba a Dios creando los cuatro reinos de la «escala de los seres naturales» separados por negros abismos que hacían imposible cualquier derivación de unos a partir de otros (fig. 1) ${ }^{21}$.

En los sesenta, aunque no se abordó abiertamente la cuestión en ningún texto de enseñanza media, encontramos esporádicas alusiones que mostraban una perspectiva evolucionista en obras de los botánicos Justo Ruiz de Azúa (1903-1980) y Emilio Guinea López (1907-1985)22.

Uno de los casos más interesantes que mostró una diferencia entre la censura en el ámbito universitario y preuniversitario lo encontramos en algunos textos de Salustio Alvarado. Aunque su Biología Preuniversitaria de 1963 señalaba la realidad de la evolución para explicar las adaptaciones de los seres vivos ${ }^{23}$, afirmaba sin embargo «oscuridades y penumbras» en las causas y

18 ANÓnimo (1943), Historia Natural, Zaragoza, Luis Vives, pp. 103-104.

19 Vicente Muedra era licenciado en Ciencias Naturales y miembro de la Real Sociedad Española de Historia Natural, fue autor de manuales para varios cursos de bachillerato, obras de 'apologética científica' (véase más adelante) y atlas de anatomía animal y humana que conocieron numerosas ediciones hasta la década de 1980. Muedra también colaboró en varias obras con los catedráticos de Paleontología, Bermudo Meléndez (1913-1999), y de Zoología, Andrés de Haro (1925-2006).

20 Muedra, V. (1956, 7. a ed.), Ciencias Naturales. Segundo curso de Bachillerato, Barcelona, Dalmau y Jover, pp. 9-14.

21 Véase el texto que acompañaba esta ilustración en el apartado 5.4 de este artículo.

22 Las expresiones «precursor del caballo» y «crustáceos antecesores de los actuales» aparecen en RUIZ dE AzÚA, J. (1963), Ciencias Naturales. 5. ${ }^{\circ}$ Curso, Vitoria, Ed. Montepío diocesano, pp. 134 y 139. Emilio Guinea López (1907-1985) habla de «realidad evolutiva» y de las relaciones entre el desarrollo embrionario y la filogenia, citando a Haeckel y Darwin: GuineA, E. (1966), Biología, Madrid, Anaya, pp. 209-216.

23 Alvarado, S. (1966), Biología Preuniversitaria, Madrid, Artes Gráficas y Ediciones, p. 247. 
mecanismos del proceso. Esto contrastaba con el tratamiento y extensión dos capítulos y cuarenta páginas - que el mismo autor había dedicado unos años antes a la evolución en su manual universitario Biología General (6. ${ }^{\mathrm{a}}$ ed., 1959), así como con el reconocimiento mostrado hacia Darwin y la teoría de la selección natural en el centenario de la publicación de El origen de las especies $^{24}$.

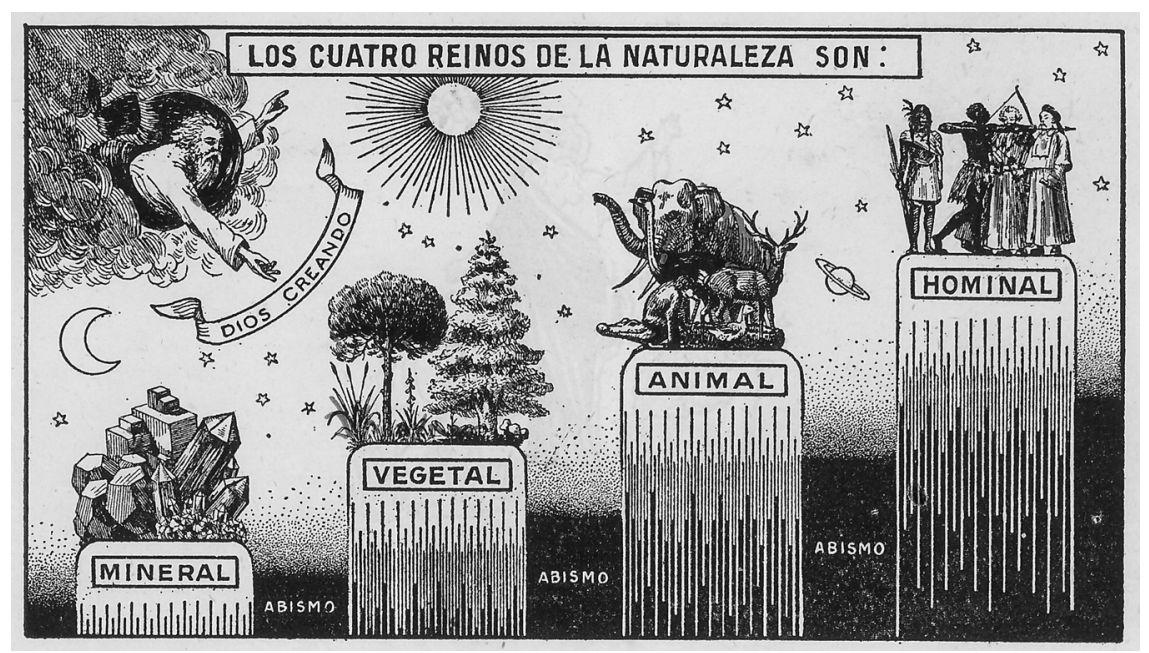

Fig. 1. Dios creando los cuatro reinos de la naturaleza. Ciencias naturales para segundo de Bachillerato, de Vicente Muedra (1956).

\section{A DIOS POR LA CIENCIA: DIVULGACIÓN}

Aparte de la educación, la biología teísta contó con una importante difusión social a través de obras de divulgación entre las que destacaron las de los jesuitas Vicente Muedra y Jesús Simón (1891-1971). La relación entre divulgación y enseñanza era muy estrecha; Muedra fue profesor de ciencias naturales en el colegio Santo Domingo de Orihuela y autor de libros de texto para

24 Alvarado, S. (1959, 6. a ed.), Biología General, Madrid, Impr. Agesa, pp. 309-348. Véase BLÁzQuEZ, F. (2007), El centenario de El origen de las especies en España (1959), eVOLUCIÓN. Revista de la Sociedad Española de Biología Evolutiva (edición electrónica), 2, pp. 33-41. Puede consultarse en: http://www.sesbe.org/sites/sesbe.org/files/eVOLUCION-02_2.pdf(29/07/2011). 
varios cursos de bachillerato, y las obras de Simón fueron lecturas recomendadas en algún centro educativo ${ }^{25}$.

Jesús Simón pronunció numerosas conferencias sobre astronomía en los cuarenta y cincuenta ${ }^{26}$ y dos de sus obras, cuyo subtítulo era Estudios cientifico-apologéticos, fueron especialmente importantes por el número de ediciones y años que abarcaron. Su obra principal, A Dios por la Ciencia ${ }^{27}$ (fig. 2) apareció en 1941 y contó con diez ediciones, la última en 1979. El prólogo a la segunda edición (1943) indicaba que su objetivo era «reducir el número de ateos» y romper con las enseñanzas y la divulgación realizadas durante el período republicano. Se trataba de una obra bien ilustrada que exponía las maravillas y la perfección de la Naturaleza en todos sus órdenes (astronomía, zoología, botánica o anatomía humana), como pruebas de la existencia, poder y sabiduría divinos. Una cita de Linneo en la portada mostraba la deuda con la teología natural predarwiniana: «He rastreado las huellas de su acción en las criaturas, y, en todas, aun en las más ínfimas y más cercanas a la nada, ¡qué poder, qué sabiduría, qué insondables perfecciones he encontrado!».

La otra obra de Simón, El Hombre ${ }^{28}$, apareció a finales de los cuarenta y su última edición fue en 1962. En ella mostraba al «hombre» - no a la mujer- como el centro de la Creación ${ }^{29}$, señalando el abismo que le separaba

25 CERDÁ, E. (2009), La biología de Dios, reseña de Ayala (2007), en Revista de Libros, 153, septiembre. El autor señala cómo sus profesores de bachillerato le indujeron a leer $A$ Dios por la ciencia del jesuita Jesús Simón.

26 Diario $A B C$, edición de Andalucía, de 17 de enero de 1956, p. 18. También se citan conferencias suyas en mayo de 1940, véase MORENO, M. (1999), La Diócesis de Orihuela-Alicante en el franquismo: 1939-1975, Tesis doctoral, Publicaciones de la Universidad de Alicante, p. 101.

27 SimÓn, J. (1941, 1943, 3. ${ }^{\mathrm{a}}$ ed. [s/f], 1947, 1950, 1954, 1958, 1961, 1969), A Dios por la Ciencia. Estudios científico-apologéticos, Barcelona, Lumen. Décima edición: Madrid, Ed. Alonso, 1979. Se ha consultado la sexta edición, a esta se corresponde la referencia SIMÓN (1954). Según el autor, en la primera edición (1941) se vendieron 4000 ejemplares y diez años más tarde ya se alcanzaban 30.000. El ministro Ibáñez Martín utilizó una consigna equivalente, «Por la ciencia hacia Dios», en un discurso en la Universidad de Valencia en 1944, véase Claret, J. (2006), El atroz desmoche. La destrucción de la Universidad española por el franquismo, 1936-1945, Barcelona, Crítica, pp. 58 y 382.

28 Simón, J. (1944, 1948, 1954 y 1962), El Hombre. Estudios científico-apologéticos sobre su origen, antigüedad, naturaleza y destino, Barcelona, Lumen. Todas las citas en este artículo pertenecen a la segunda edición de esta obra: SIMÓN (1948).

29 Podría pensarse que 'hombre' es un término genérico para designar a la especie humana pero Simón expresaba claramente que sólo el «primer Padre» es «el origen único de todo el linaje humano» pues, según el relato del Génesis, «la mujer procede del hombre», lo que le servía para extraer lamentables consecuencias acerca de su papel en la familia y en la sociedad. SIMÓN (1948), pp. 125-128. 
del mono, argumentando contra el evolucionismo y censurando incluso a los autores católicos que lo defendían; cerraban la obra varios capítulos dedicados a la naturaleza e inmortalidad del alma humana.

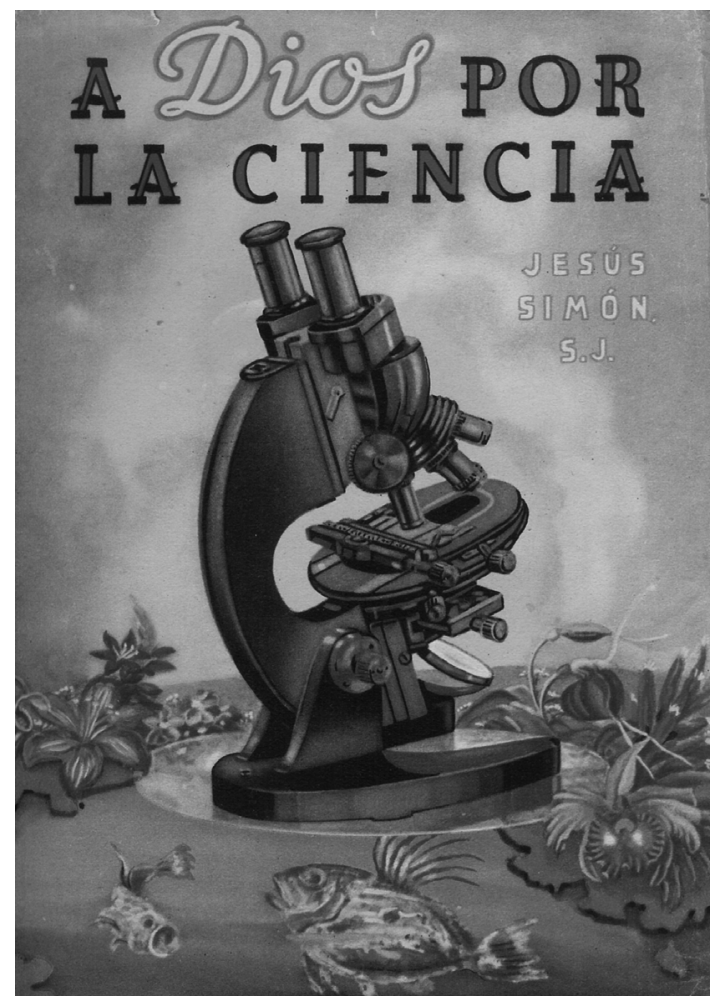

Fig. 2. Cubierta de la obra de Jesús Simón, A Dios por la ciencia. Estudios científico-apologéticos (edición de 1954).

A finales de los cuarenta apareció La perfección científica en las obras animales $^{30}$, del ya citado Vicente Muedra (fig. 3), inscrita en lo que el autor

30 Se ha consultado Muedra, V. (1948, 2. ${ }^{\mathrm{a}}$ ed.), La perfección cientifica en las obras animales. Narraciones científico-recreativas (primera serie), Murcia, Nogués. No hay datos sobre la primera edición aunque alguna librería de viejo la fecha el mismo año que la segunda. El autor también publicó un folleto de idéntico título en 1956 (Barcelona, Dalmau Socías). 


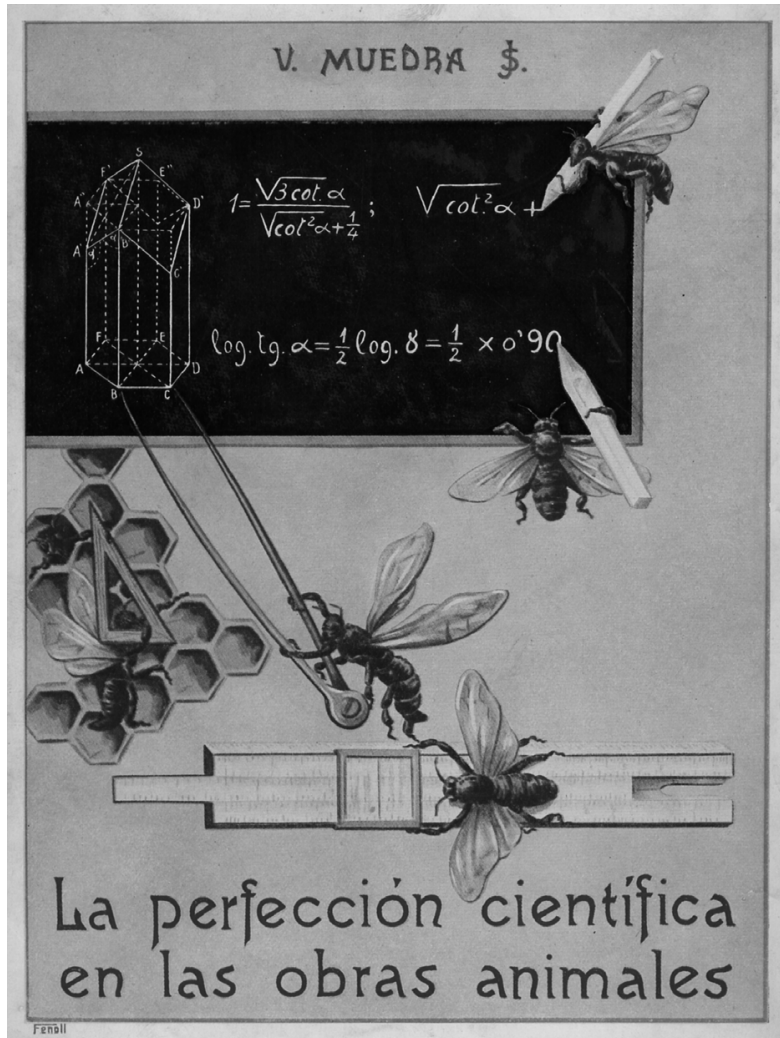

Fig. 3. Cubierta de La perfección cientifica de las obras animales de Vicente Muedra (1948).

denominó Narraciones científico-recreativas y a las cuales pertenecieron varias obras de menor extensión que se publicaron a lo largo de los cincuenta también con notable éxito ${ }^{31}$. La perfección científica... estaba especialmente dirigida a «jóvenes estudiosos» y su objetivo fundamental era, aparte de «ver

31 Muedra, V. (1950), Maravillas cientificas en los actos de los animales. Narraciones científico recreativas, vol. IV, Barcelona Tip. Cat. Casals. MuedrA, V. (1952), La ciencia al servicio de los animales. Narraciones científico-recreativas, vol. V, Barcelona, Tip. Cat. Casals. MuedrA, V. (1954), El maravilloso instinto de los animales. Narraciones científicorecreativas, vol. VI, Barcelona, Tip. Casals. MUEDRA, V. (1955), Instinto... sí! Inteligencia... no! Narraciones cientifico-recreativas, Dalmau y Jover, 1955. 
al CREADOR en las admirables obras de sus criaturas», mostrar cómo los animales habían resuelto multitud de problemas en la naturaleza a través de comportamientos (instintos) que eran la manifestación de la sabiduría y ciencia del ser superior que los había creado. Entre los numerosos ejemplos de esta obra destacaban las formas de defensa animal en cuya descripción el autor utilizaba normalmente metáforas bélicas.

Por último, a finales de los cincuenta, casi coincidiendo con el centenario de la publicación de El origen de las especies, Muedra publicó el primer tomo de otra obra que también divulgaba un pensamiento creacionista, La Naturaleza $(1958)^{32}$. El comienzo era claramente apologético e incluía el pasaje del Génesis, el resto era una descripción de los distintos grupos zoológicos, sin alusiones religiosas, con un estilo claro y unas ilustraciones a color que la convertían en una obra amena para un público infantil y juvenil.

\section{TEOLOGÍA NATURAL EN EL FRANQUISMO: ARGUMENTOS, ICONOS Y FOBIAS}

\subsection{La palabra del Génesis}

El primer pasaje del Génesis, en el que se relata la creación del mundo, fue reproducido y comentado en las obras de ciencias naturales de enseñanza y divulgativas que defendieron la perspectiva creacionista ${ }^{33}$. El texto se insertaba normalmente en las primeras páginas poniendo de manifiesto la supeditación del discurso científico al religioso ${ }^{34}$.

Los primeros capítulos del Génesis, según había dictaminado la Pontificia Comisión Bíblica de $1909^{35}$, seguían considerándose históricos. Como afirmaba Jesús Simón: «la palabra de Dios no yerra. Lo que es deficiente, muchas veces, es nuestro conocimiento de ella, nuestra precipitación y poca

32 Muedra, V. (1958), La Naturaleza, tomo I: Invertebrados, Barcelona, Dalmau y Jover. La editorial recibió el premio Lazarillo por esta obra en 1959, debido a su cuidada edición y magníficas ilustraciones. Véase BERMEJo, A. y Figuerola, M. (2000), Editoriales premiadas en Lazarillo, Revista de la Asociación de Amigos del Libro Infantil y Juvenil, 2, p. 50.

33 Muedra (1956), p. 14; Muedra (1958), pp. 5-6; TABOAS, J. (aprox. 1958), Historia Natural y su metodología, Madrid, Impresos Marcos, pp. 7-13.

34 Muedra (1956), p. 13. También Muedra (1958), p. 6

35 Pontificia COMisión BíBlica (1909), Sobre el carácter histórico de los tres primeros capitulos del Génesis (30 de junio de 1909). Puede consultarse en: http://www.vatican.va/ roman_curia/congregations/cfaith/pcb_doc_index_sp.htm 
ciencia ${ }^{36}$. Aunque en numerosas ocasiones se indicó que este relato no era científico, lo cierto es que su interpretación delimitaba aquello que podía ser admitido por la ciencia desde la perspectiva católica. En El Hombre (Simón, 1948) la narración de los acontecimientos geológicos y biológicos de la historia de nuestro planeta se hacía en perfecta concordancia con el texto bíblico, incluso la tardía creación del Sol, la Luna y las estrellas, el cuarto día -después de la creación de la Tierra-, no planteaba ningún problema pues su autor lo interpretaba como la visión por vez primera de estos astros, tras la liberación de la densa capa de anhídrido carbónico de la atmósfera ${ }^{37}$.

El único aspecto sujeto a una cierta flexibilidad de interpretación fue la duración de los «días de la Creación», algo sobre lo que la Comisión Bíblica de 1909 ya había dado libertad de discusión. Dadas las evidencias acumuladas en torno a la antigüedad de la Tierra, los autores creacionistas consideraron que se trataban de lapsos de tiempo indefinido, de miles o millones de años, y puesto que la $\mathrm{Bi}$ blia no establecía cronología alguna, este problema quedaba «a las discusiones de los hombres y a las investigaciones de la Ciencia» ${ }^{38}$, lo que era un reconocimiento más de que la ciencia sólo podía ocupar el espacio que dejaba la Biblia.

Uno de los casos más interesantes que reveló un cambio en la consideración del texto bíblico a lo largo de una década, lo observamos en la obra divulgativa La Naturaleza de Vicente Muedra. La edición de 1958 se iniciaba con un prólogo que, en mayúsculas, afirmaba el origen común de todos los seres era «la Creación» y reproducía el pasaje del Génesis ilustrado con la escala natural culminada por el hombre. El prólogo y el pasaje - no la ilustración - desaparecieron en la edición de 1969, siendo sustituidos por un prefacio del paleontólogo Bermudo Melénde ${ }^{39}$ que indicaba «el hecho innegable de que los seres vivos han variado en el tiempo» y que, aunque el Génesis no era un libro científico, su mensaje era perfectamente válido: la Naturaleza era obra de Dios y ello no se oponía a una concepción evolutiva de la misma sino que mostraba una «Inteligencia infinita, que, desde la iniciación de los fenómenos vitales en la Tierra, tenía ya prevista su culminación en el mundo vivo actual $»^{40}$.

\footnotetext{
36 SiMÓN (1948), p. 36.

37 SiMÓN (1948), pp. 40-41.

38 SiMÓN (1948), p. 16.

39 Sobre el evolucionismo de Bermudo Meléndez puede consultarse BLÁZQUEZ (2001), pp. 301-303 y Pelayo (2009), Debatiendo sobre Darwin en España: Antidarwinismo, teorías evolucionistas alternativas y síntesis moderna, Asclepio, 61 (2), pp. 117-122.

40 Prólogo de Bermudo Meléndez a MuedrA, V. (1969), La Naturaleza, tomo I: Invertebrados, Barcelona, Jover, pp. 5-6.
} 


\subsection{Creación y generación espontánea. El icono Pasteur}

En España, las publicaciones divulgativas o didácticas de los cuarenta y cincuenta mostraron únicamente dos posibilidades en torno al origen de la vida: «generación espontánea, que excluye toda intervención divina... o un acto especial de Dios Creador ${ }^{41}$, indicando siempre que no había término medio y eludiendo (o desconociendo) las aportaciones que habían tenido lugar con los trabajos de Alexander I. Oparin (1894-1980), durante el primer tercio del siglo XX, o los experimentos de 1953 de Stanley L. Miller (1930-2007) ${ }^{42}$.

La teoría de la generación espontánea ya había sido desmontaba por los experimentos de Louis Pasteur (1822-1895), que se convirtió en un magnífico icono científico para la nueva biología de posguerra y de quien se publicaron numerosas biografías, siendo frecuentes las ilustraciones con su figura y las citas que se referían a él como «sabio y católico», «insigne bienhechor» y «Santo» ${ }^{43}$.

En realidad, Pasteur era el sustituto perfecto de quien había sido icono de la biología republicana, Charles Darwin, a quien un catecismo (antes y después de la guerra) consideraba un «pretendido sabio... dotado por Dios de gran espíritu observador pero de muy poca inteligencia» ${ }^{44}$. Tras la Guerra Civil se evitaron las alusiones a Darwin en los textos de ciencias naturales y, salvo alguna excepción, sus obras desaparecieron de las librerías y bibliotecas hasta principios de los sesenta ${ }^{45}$.

41 Muedra (1958), p. 7. En idénticos términos se expresa en SimÓn (1954), p. 102.

42 La primera referencia a estos trabajos la hemos hallado en el suplemento de la Enciclopedia Ilustrada Espasa Calpe de 1957-1958, pp. 332-336, a cargo de Rafael Alvarado (1924-2001) que actualizaba la cuestión en España y añadía que las interpretaciones materialistas sobre el origen de la vida no atentaban contra la ortodoxia católica.

43 Véase Muedra (1956), p. 271 y Muedra (1958), p. 8. Entre las biografías de Pasteur en la posguerra destacaron: Lleonart, J. (1943), Vida de Luis Pasteur, Barcelona, Seix Barral Hnos.; SALÓ, A. (1944), Pasteur, Barcelona, Felipe Glez.-Rojas, y PASTEUR, L. (1945), Biografia, s. 1., Ed. España.

44 Márquez, G. (1929), Explicación literal del Catecismo de Ripalda con una exposición y refutación de los errores modernos y la explicación de la bula de la Santa Cruzada, Razón y fé. Madrid, p. 347. Igualmente presente en la edición de 1951 reproducida por MIRET y SÁDABA (1998), p. 213.

45 Las excepciones fueron dos ediciones del relato del viaje del Beagle y DARWIN, C. (1950), El origen de las especies por selección natural, Madrid, Ed. Ibéricas. Véase GoMIS, A. y JosA, J. (2008), Bibliografia crítica ilustrada de las obras de Darwin en España (18572008), Madrid, CSIC. 


\subsection{Retorno al pasado}

La visión decimonónica del problema del origen de la vida fue un ejemplo más del retorno a debates biológicos del pasado en la divulgación y enseñanza durante el franquismo. Para proclamar la muerte del darwinismo no hizo falta volver la vista muchos años atrás pues, durante las tres primeras décadas del siglo XX, la teoría de la selección natural sufrió un importante descrédito en el ámbito científico. En la dictadura, muchos textos incluyeron citas y argumentos de biólogos antidarwinistas de aquel período para proclamar la defunción científica de la teoría de la evolución y considerar al darwinismo «un tronco viejo y carcomido» ${ }^{46}$.

Historia Natural y su metodología ${ }^{47}$, una obra dirigida a los alumnos que iniciaban sus estudios de Magisterio, no pudo expresarlo más claramente. En su primera página, con el título «La creación» y en fechas próximas al centenario de El origen de las especies, encontramos:

En plena decadencia y próxima a su ocaso la teoría EVOLUCIONISTA sobre el origen de las especies, prohibida su defensa en muchos países cultos como teoría sin base científica, por carecer de pruebas concluyentes que la confirmen... estudiada desapasionadamente, sólo desde el punto de vista científico, sin los prejuicios anticatólicos del profesor alemán Ernesto Haeckel y sus seguidores, que pretendían encontrar en la teoría de la evolución «la artillería pesada contra la Iglesia», pronto se le encontraron sus «puntos débiles» y la «difícil demostrabilidad de sus teorías», estando en la actualidad, como hemos dicho, en plena decadencia y pronto a desaparecer, quedando subsistente, con todo su esplendor, la necesidad de admitir la existencia de un Ente necesario, de Dios, causa y origen de todo lo que existe, que ha dado principio a todas las cosas, a cuya acción creadora, por su Omnipotencia, se debe la presencia de todos los seres naturales, contingentes, alterables y caducos, que en infinita variedad pueblan el Universo ${ }^{48}$.

El intento de conciliar la historia de la Tierra con los episodios descritos en el Génesis también supuso una vuelta a debates geológicos del XIX. Incluso el mito de la Atlántida fue considerado por el jesuita Simón como puente intercontinental para explicar algunos datos sobre la distribución de las espe$\operatorname{cies}^{49}$. Y mucho más representativo de esta vuelta al pasado fue el rescate de

46 Palabras del zoólogo alemán Oskar Hertwig (1849-1922) según Simón (1948), p. 7.

47 TABOAS, J. (aprox. 1958), Historia Natural y su metodología, Madrid, Impresos Marcos.

48 TABOAS (aprox. 1958), p. 7. Se han respetado las mayúsculas del texto original.

49 SimÓN (1948), p. 23. Véanse las ideas biogeográficas de Edward Forbes (1815-1854) que implicaban la existencia de la Atlántida en MAYR (1982), p. 144. Algunos debates a este 
la teoría de las creaciones sucesivas de George Cuvier (1769-1832) y Alcide D’Orbigny $(1802-1857)^{50}$ :

La doctrina de las CREACIONES SUCESIVAS explica la sucesiva aparición de los seres en distintos períodos geológicos por cambios que determinaron distintas condiciones para la vida orgánica de un período a su inmediato subsiguiente, desapareciendo unas especies y apareciendo otras por la intervención creadora de Dios, acción creadora que se repitió en el comienzo de cada época hasta la aparición del hombre, que fue la última manifestación creadora del Supremo Hacedor ${ }^{51}$.

Paradójicamente, estas exaltaciones antievolucionistas que tenían lugar en España ocurrían cuando la síntesis evolucionista se estaba gestando o había sido ya plenamente aceptada por la biología. Este hecho fue silenciado en los manuales de enseñanza media, y rebatido en publicaciones divulgativas y académicas $^{52}$ hasta finales de los años cincuenta, momento en el que el evolucionismo darwinista comenzó a ser recuperado con motivo del centenario de la publicación de El origen de las especies.

5.4. El círculo de hierro de la especie y los abismos impenetrables de la escala natural

Los autores defensores de la biología creacionista tenían un concepto esencialista de especie biológica. Según esta visión, las especies eran entidades inmutables, constituidas por un grupo de características definitorias, y las variaciones respecto de su 'esencia' eran 'desviaciones' o modificaciones debidas a las condiciones ambientales. Esta concepción prevaleció durante la mayor parte de la historia de la biología hasta el desarrollo de la teoría de la selección

respecto tuvieron lugar en casa de Darwin en 1845, véase Boulter, M. (2009), Darwin's Garden, London, Constable, p. 132.

50 Véase el capítulo "Geología bíblica y creacionismo: una alternativa frente a las propuestas materialistas» en Pelayo, F. (1999), Ciencia y creencia en España durante el siglo $X I X$, Madrid, CSIC, pp. 47-80.

51 TABOAS (aprox. 1958), p. 7. Se han respetado las mayúsculas del texto original.

52 Sobre la teoría sintética en España y la evolución en el franquismo véase BLÁZQUEZ, F. (2001), La teoría sintética de la evolución en España. Primeros encuentros y desencuentros, Llull, 50 (24), pp. 289-313; y Pelayo, F. (2009), pp. 101-128. 
darwiniana que señaló la importancia de la variación dentro de la especies en el proceso de selección natural ${ }^{53}$.

El concepto esencialista de especie era coherente con el creacionismo expresado en el Génesis, y el significado y papel de la variación se entendía de la siguiente forma: «Dios ha concedido sabiamente cierto ámbito a cada especie para que pueda desenvolverse con amplitud... pero todo ello dentro del círculo de hierro de la especie, de la cual no pueden salir» ${ }^{54}$. Cada especie era pues «una unidad fija creada por Dios, con una esencia propia e incomunicable, con organización y aun células propias que no admiten mezcla alguna» ${ }^{55}$.

Este esencialismo era aplicado además a todo el mundo orgánico donde «abismos impenetrables» separaban los reinos que componían la escala de los seres naturales. Esta concepción de la naturaleza quedó magníficamente expresada en la ilustración de la figura 1 y el texto que la acompañaba en el manual de ciencias naturales de Vicente Muedra (1956):

En el escalón inferior están los seres inorgánicos: minerales y rocas; puesto que son los seres más inferiores de la creación.

En el escalón inmediato superior están los vegetales, formando un grupo aparte sin comunicación con los de abajo, ni con los de arriba; sólo son superiores a los del grupo anterior.

En el escalón inmediato superior están los animales, formando también ellos solos su grupo independiente, mediando un abismo entre ellos con el grupo de abajo de quienes son superiores y otro abismo impenetrable, con los del grupo de arriba, de quienes son inferiores.

Finalmente, en el escalón superior, y ocupando la cúspide de toda esta escalera, se encuentra el hombre, dominando a todos los demás seres, como REY DE LA CREACIÓN.

Es imposible que los del escalón inferior, es decir, los animales, puedan llegar adonde está el hombre, porque el abismo que separa estos dos grupos, es impenetrable y no puede construirse puente o lazo de unión ninguno que los acerque: y menos, que los una.

El hombre tiene un alma inmortal e inteligente que ni tiene ni podrá tener jamás el animal: He aquí el abismo que separa ambos grupos y que los mantendrá siempre distantes entre sí. Por esta razón, el hombre tiene inteligencia. En cambio, el animal, no tiene inteligencia, y por eso, ni discurre ni progresa ${ }^{56}$.

53 Véase MAYR (1992), pp. 53-55.

54 SiMÓN (1948), p. 108.

55 SIMÓN (1948), p. 108.

56 Muedra (1956), p. 13. 


\subsection{El hombre: origen, naturaleza y diferencias con el mono}

A pesar de que durante el primer tercio del siglo XX se promovieron líneas de investigación en evolución humana ligadas a la Junta de Ampliación de Estudios $^{57}$, la Guerra Civil y la dictadura provocaron un retroceso lamentable para la paleontología humana en España ${ }^{58}$. Para la biología teísta el ser humano fue creado a imagen y semejanza de Dios, era un «ángel humanado» y con frecuencia se señalaba su carácter divino y su condición — normalmente en mayúsculas - de «REY DE LA CREACIÓN».

En los textos religiosos del franquismo se solía recordar que la creación divina del ser humano era un dogma de fe del Concilio de Letrán IV (siglo XIII) y que los teólogos y los Santos Padres interpretaban las palabras de la Sagrada Escritura como una acción directa de Dios en la creación del hombre, «luego es temerario separarse de este sentido, y, por tanto, debemos afirmar que Dios formó el cuerpo del primer hombre» ${ }^{59}$. Dejando claro que este «no procede, por evolución o transformación de la materia y de las especies, de otros animales menos perfectos» ${ }^{60}$. La creación de la primera mujer se consideraba un episodio secundario pues, siguiendo literalmente el relato del Génesis, provenía «de una parte del hombre por modo maravilloso, a manera como de las simientes y de las raíces hace crecer las plantas» ${ }^{61}$.

$\mathrm{Al}$ considerar al ser humano (en realidad, al «hombre») rey de la Creación, la historia de la Tierra adquiría un carácter finalista: «Nuestra morada ha ido cambiando de faz, renovándose, ensayándose dijéramos, para recibir, al fin y ofrecer digno palacio al ser privilegiado que había de ennoblecerla sobre todos, el hombre» ${ }^{62}$.

$\mathrm{Al}$ margen de los problemas relacionados con la exégesis bíblica o la naturaleza del pecado original, el origen animal de los seres humanos planteaba un nuevo inconveniente relacionado con nuestra posición en la Naturaleza. Inclui-

57 Véase Pelayo, F. (2007), La evolución humana y su difusión en España en el marco de la JAE (1907-1939), Asclepio. Revista de Historia de la Medicina y de la Ciencia, 59, (2), pp. 137-162.

58 Véase a este respecto IGLESIAS, A. (1998), A investigación en España sobre o evolucionismo humano (1936-1976), Tesis Doctoral, Santiago de Compostela, Universidad de Santiago de Compostela.

59 Villapún (1949), pp. 55 y 59.

60 AAVV (1959), Contestaciones al cuestionario oficial de las oposiciones de ingreso en el Magisterio Nacional, Madrid, Escuela Española, pp. 24-25.

61 SimÓN (1948), pp. 125-128. Véase nota 29.

62 SIMÓN (1948), p. 20. De forma idéntica podemos encontrarlo en MuEDRA (1956), p. 304. 
dos en el orden Primates desde la clasificación de Linneo en el siglo XVIII, fueron numerosas las obras de ciencias naturales durante el franquismo en las que se omitió este hecho; había un lugar para orangutanes, chimpancés y gorilas entre los primates antropomorfos, pero los humanos quedábamos fuera de cualquier clasificación ${ }^{63}$. Se aceptaba que «aunque el ser humano es un mamífero» era un error incluir su estudio entre ellos «como si fuera uno de tantos», afirmaba Vicente Muedra, debido a la presencia de alma inteligente e inmortal que nos separaba de los animales y nos acercaba a los ángeles y a Dios. Por esta razón, se aceptó en algunos casos la existencia de un suborden Bimanos ${ }^{64} \mathrm{y}$ en otros un reino aparte, el reino Hominal, tal y como habían defendido algunos médicos y naturalistas españoles en el siglo XIX ${ }^{65}$.

Esta separación del resto de la Naturaleza llevaba frecuentemente a señalar en las obras las diferencias (físicas, intelectuales y morales) entre el hombre y el mono (o los antropomorfos), añadiendo en algún caso que: «Junto a estos caracteres conviene tener presente la gallardía de las formas en el hombre, la majestad de su porte, la elocuencia de su mirada, etc., que caracterizan al "rey de la Creación"» ${ }^{66}$.

En este sentido, la versión católica del evolucionismo que aceptaba la intervención divina en este proceso fue mirada con recelo y criticada en obras de divulgación. Así se refería el padre Simón al biólogo católico George J. Mivart (1827-1900), que aceptó el origen animal del cuerpo humano a finales del XIX: «La opinión de Mivart, pues, no es condenable, pero ¿es defendible? ¿Está conforme con el texto bíblico? Evidentemente que no... ¿Qué razones aducen Mivart y sus partidarios para justificar su teoría? Exactamente las mismas que los darvinistas» ${ }^{67}$.

\subsection{Fisiología vitalista contra la biología materialista}

Al lado de los problemas que generaba el apego a la literalidad del Génesis en lo referente al origen de los seres vivos y del ser humano, había un problema de mucha mayor trascendencia que ya se había señalado antes de la

63 Véase por ejemplo Álvarez, E. y Mingarro, A., Elementos de Ciencias de la Naturaleza II, Madrid, Summa, 1945.

64 ANÓNIMO (1943), Historia Natural, Zaragoza, Luis vives, pp. 357-358.

65 Gomis, A. (2004), El reino Hominal: un ejemplo de ideología frente a ciencia en la España del siglo XIX, Llull, 27, pp. 423-434.

66 ANÓNIMO (1943), p. 358. También se expresan las diferencias en VILLAPÚN (1949), p. 60.

67 SIMÓN (1948), pp. 120-122. 
Guerra Civil: el materialismo. Para el catecismo Ripalda en su edición de 1929 y el Nuevo Ripalda en la Nueva España (1951), los dos primeros errores del mundo moderno eran el materialismo y el darwinismo, por delante del ateísmo, el deísmo, el socialismo, etcétera ${ }^{68}$.

La concepción materialista y evolutiva de la vida resolvía de forma natural el problema de los «abismos impenetrables» entre lo vivo y lo inerte, entre unas especies y otras o entre los animales y los seres humanos. Pero además el materialismo biológico negaba o eludía el problema del cuerpo y el alma, que en definitiva era un caso particular de la gran dualidad entre el mundo natural y el sobrenatural. En muchos textos de ciencias naturales, al inicio de las descripciones anatómicas y fisiológicas, se indicaba que «el hombre» estaba compuesto por «cuerpo y alma racional» y que las ciencias naturales solamente se ocupaban del primero ${ }^{69}$.

Por otra parte, la biología en el primer tercio del siglo XX ya había rechazado la existencia de fuerzas vitales para explicar la fisiología de los seres vivos, aceptando que las ciencias físicas y químicas podían dar cuenta plenamente de los fenómenos biológicos. Contra esta biología materialista, renace en el franquismo una fisiología vitalista - ya presente antes de la Guerra Civil- que señalaba la insuficiencia de las explicaciones físico-químicas y la existencia de fuerzas distintas a las conocidas. Incluso la fisiología de los vegetales fue interpretada de esta forma, así el geotropismo de la raíz y el tallo o el ascenso del agua en los árboles eran considerados por el padre Simón «un enigma, un misterio incomprensible» para la ciencia materialista, pues «en las plantas, lo mismo que en los animales, no todo puede ser explicado ni química ni físicamente; actúan en ellas fuerzas que son, evidentemente, de otra índole, fuerzas superiores que rigen y se imponen aunque no aparezcan visibles $\rangle^{70}$.

\subsection{Armonía, orden, providencia, diseño y azar}

Al igual que la teología natural del siglo XIX, la biología teísta del franquismo expuso - aunque de una forma mucho más simple-, que el orden y

68 Véase MÁrquez, G. (1940, 5. ${ }^{\mathrm{a}}$ ed.), Explicación literal del nuevo catecismo de Ripalda, Madrid, Razón y Fe, pp. 267-276. Esto continuaba en la edición de 1951, reproducida por Miret y SÁDABA, 1998.

69 Por ejemplo: Anónimo (1954), Ciencias Naturales. Segundo curso de Bachillerato, Zaragoza, Luis Vives.

70 Simón (1954), pp. 256 y 291. 
armonía del mundo encerraban a su vez varios órdenes: el astronómico, el de la Naturaleza (cuyo lugar supremo ocupaba el hombre) y el de la perfección humana expresada en su anatomía y fisiología ${ }^{71}$.

En A Dios por la ciencia se exponía cómo la grandeza de la arquitectura y armonía del universo suponían la existencia de una causa omnipotente e infinita que sacó a los astros de la nada y que, al mismo tiempo, era el origen del movimiento de las inmensas aglomeraciones de materia ${ }^{72}$.

El orden en la Naturaleza se percibía en la jerarquía de los seres vivos, la Scala Naturae coronada por el ser humano (fig. 1) ${ }^{73}$, y también en un orden ecológico que mostraba a los seres interconectados en ciclos de materia y energía, así las plantas absorben el dióxido de carbono generado por los animales en la respiración y ello era considerado otro ejemplo de sabiduría y finalidad divinas: «¿Será todo casual? El materialismo dirá que sí, pero la razón y el sentido común y la Ciencia, dicen lo contrario» ${ }^{74}$.

Considerados como especies o individuos, los seres vivos también parecían mostrar un diseño inteligente. En este sentido, la complejidad de un órgano como el ojo seguía ejerciendo la misma fascinación en nuestra posguerra que en los tratados de teología natural del siglo XIX:

¿... podrá alguien creer sinceramente que todo lo que llevamos expuesto [morfología y estructura del ojo] es obra de la casualidad? Y descendiendo más a lo particular, ¿puede alguien que se persuada de que el cristalino, por ejemplo, con sus millones de fibras refringentes, que la retina con sus miríadas de conos y bastoncitos, que las capas envolventes del ojo, la córnea con el iris, los lacrimales, los músculos del movimiento, los párpados se han formado al azar sin que ninguna inteligencia sabia los haya ideado y una mano omnipotente construido? ${ }^{75}$.

El ojo no fue el único ejemplo coincidente con la teología natural decimonónica, también la metáfora del reloj, expuesta por William Paley en 1802, seguía teniendo vigencia. En A Dios por la ciencia del padre Simón, encontramos:

Nosotros tomamos en nuestras manos un mecanismo complicado, un magnífico reloj, por ejemplo, o un máquina de escribir: examinamos su elegante forma exterior,

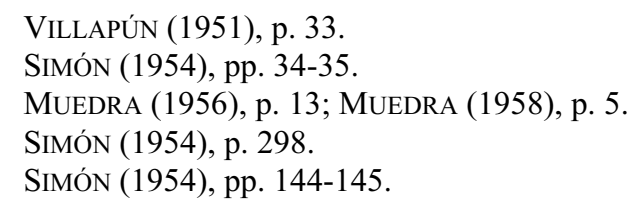


notamos sus inauditas complicaciones, sus ruedas y engranajes, la exactísima trabazón y destino de la diversas piezas, las cuales no son solamente una filigrana en sí mismas sino que concurren harmoniosamente a un fin determinado, y no dudamos, no podemos dudar, de que aquello no es obra del acaso, sino efecto sazonado de la industria y del ingenio humano, destello magnífico de la inteligencia del hombre.

Es el Ser Sabio y Omnipotente que ha enlazado las partes y los seres todos del Universo como un inteligente relojero las piezas de un reloj, después de haber hecho de cada una de ellas una obra portentosa y acabada, la más estupenda filigrana ${ }^{76}$.

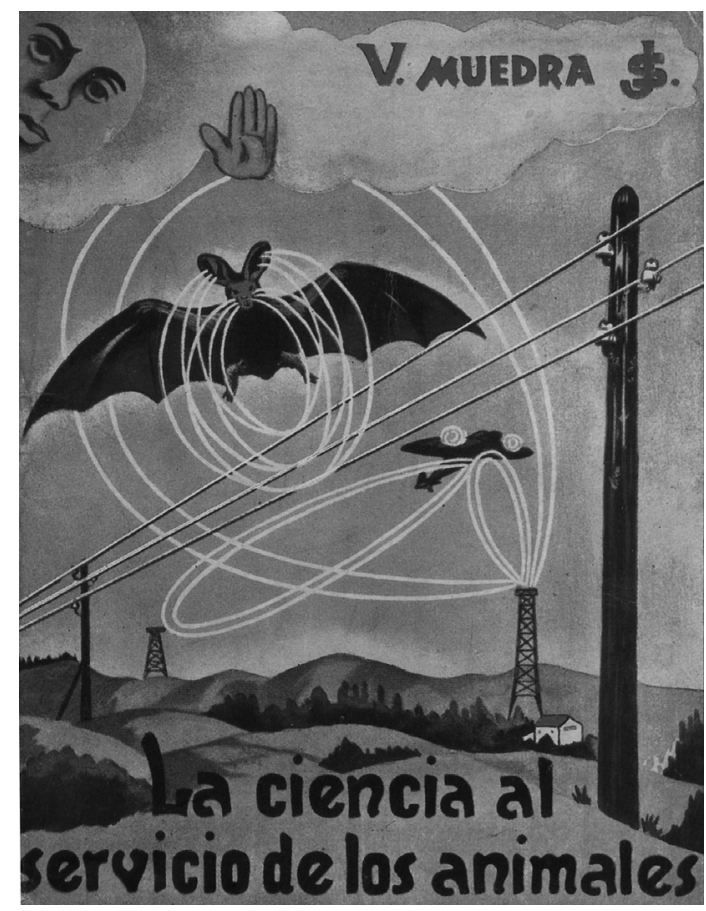

Fig. 4. Cubierta de La ciencia al servicio de los animales. Narraciones científico-recreativas de Vicente Muedra (1952).

El diseño de los seres vivos no era simplemente anatómico o fisiológico. Cuando los sacerdotes Muedra y Simón afirmaban que los «insignificantes

76 SiMÓN (1954), p. 311. 
animalillos» daban lecciones de «ciencia a nuestros ingenieros arquitectos naturalistas y fabricantes» o que «los insectos no tienen inteligencia, pero suponen la de Dios $>{ }^{77}$, expresaban que el instinto animal había sido otorgado por Dios para resolver los problemas biológicos y era muestra de su sabiduría y ciencia perfectas ${ }^{78}$. Las ilustraciones de las cubiertas de La perfección científica en las obras animales y La ciencia al servicio de los animales ${ }^{79}$ mostrando, respectivamente, afanosas abejas ingenieras tomando medidas y resolviendo los problemas matemáticos de la construcción de sus celdillas, y el murciélago, detectando los hilos eléctricos al igual que el radar humano detectaba aviones enemigos, con la vigilancia, tutela y diseño de un Dios humanizado en el cielo, son el mejor ejemplo de la iconografía de esta biología del diseño teológico (figuras 3 y 4 ).

\section{SigNOS DE CAMBIO}

Como se ha visto, en las dos primeras décadas de la dictadura la biología teísta y creacionista dominó el panorama divulgativo y la enseñanza media en España. Sin embargo, en los sesenta y principios de los setenta esta situación comenzó a cambiar.

En la divulgación, las obras descritas empezaron a coexistir con otras que mostraban un pensamiento biológico muy distinto: obras defensoras del evolucionismo finalista de Pierre Teilhard de Chardin (1881-1955) ${ }^{80}$, sobre el evolucionismo darwinista o neodarwinista ${ }^{81}$, sobre la naturaleza animal del

77 SiMÓN (1954), p. 315.

78 Véase la introducción y las conclusiones en MuEdRA (1954), pp. 5-9 y 195-203.

79 Muedra (1954) y Muedra (1952).

80 En esta divulgación destacó especialmente el paleontólogo Miquel Crusafont (19101983). En Truyols, J. (1985), Miquel Crusafont Pairó (1910-1983), Actas de la Real Sociedad Española de Historia Natural, 81, pp. 37-55; véase especialmente el número monográfico AAVV (1994) Arraona, Revista d'Història, 14 (primavera). BLÁzQUEZ, F. (2004), Entre Darwin y Teilhard. Notas sobre paleontología y evolucionismo en España (1939-1966). En Miscelánea en homenaje a Emiliano Aguirre. Paleontología, vol. II, Alcalá de Henares, Museo Arqueológico Regional, pp. 97-107; PELAYo (2009), pp. 122-126.

81 En 1963 volvieron a editarse las obras biológicas de Darwin, Gomis y Josa (2008). Véanse los trabajos originales de Faustino Cordón (1909-1999): CoRdón, F. (1958), Introducción al origen y evolución de la vida, Madrid, Taurus; CORDón, F. (1966), La evolución conjunta de los animales y su medio, Barcelona, Península. Cordón también tradujo obras de autores de la síntesis evolucionista: DobZhAnsky, T. (1955), Genética y el origen de las 
ser humano ${ }^{82}$ e incluso con obras que defendían interpretaciones materialistas de la biología y del origen de la vida ${ }^{83}$.

En este contexto divulgativo, las ediciones de A Dios por la Ciencia se distanciaron en el tiempo y su número descendió respecto de los cuarenta y cincuenta, aunque hubo una tardía edición de esta obra en $1979^{84}$.

Las obras del jesuita Muedra en los setenta fueron mucho más descriptivas al tratarse de atlas de anatomía humana y animal que llegaron incluso a la década de los ochenta. En Los animales (Muedra, 1979) ya no aparecen consideraciones religiosas y algunos párrafos mostraban un cambio radical en el pensamiento de este autor, por ejemplo, al afirmar que los equinodermos se «han originado» a partir otras formas primitivas o que las aves fósiles, como Archeopterix, poseían dientes y otras características que las «relacionan» con los reptiles ${ }^{85}$.

En la enseñanza media, que había sido escenario de silencio en torno a la evolución y, en algunos casos, de exaltación de la biología creacionista, también se produjeron cambios notables. La Ley General de Educación (4 de agosto de 1970), desarrollada a lo largo de la década de 1970, contempló una perspectiva evolucionista en las materias de Biología y Ciencias Naturales, en dos niveles distintos, algo que no ocurría desde los tiempos de la Segunda República $^{86}$. En 1975, unos meses antes de la muerte del general Franco, los cuestionarios oficiales de Religión ya no abordaban la enseñanza del Antiguo Testamento, y los de Ciencias Naturales del primer curso de aquel nuevo ba-

especies, Madrid, Revista de Occidente; MAYR, E. (1963), Especies animales y evolución, Barcelona, Ariel; SiMPSON, G. G. (1967), La vida en el pasado. Una introducción a la Paleontologia, Madrid, Alianza.

82 Morris, D. (1968), El mono desnudo. Un estudio del animal humano, Barcelona, Plaza y Janés; y MORRIS, D. (1970), El zoo humano, Barcelona, Plaza y Janés.

83 Monod, J. (1971), El azar y la necesidad. Ensayo sobre la filosofia natural de la biología moderna, Barcelona, Barral; OpARIN, A. (1970), Origen de la vida sobre la Tierra, Madrid, Tecnos.

84 Véanse las referencias a las ediciones en la nota 27. La edición de 1979 era la décima, en ella no se indicaban cambios respecto de la de 1969 y contaba con los mismos prólogos y permisos eclesiásticos. Esta obra llevaba los argumentos de diseño divino a fenómenos biológicos como la mitosis y contenía errores notables, entre ellos que el ser humano tiene 48 cromosomas (como se pensaba hasta 1956) o que los genes son «unos granitos insignificantes... espolvoreados a manera de abundante y finísimo polen» (SIMÓn [1979], p. 184).

85 Muedra (1979, 4. ${ }^{\mathrm{a}}$ ed.), Los animales, Barcelona, Jover, pp. 30, 50 y 58. Este cambio ya se observaba con las modificaciones que este autor introdujo en el prólogo a La Naturale$z a$, edición de 1969. Véase el epígrafe 5.1.

86 Véase nota 15. 
chillerato contenían un epígrafe sobre la evolución y el origen del ser humano, incluyendo entre sus objetivos didácticos «el estudio del origen desarrollo y evolución de los seres vivos que sobre la Tierra han existido y viven en la actualidad ${ }^{87}$. La reforma también afectó a cursos superiores de la enseñanza media incluyéndose en el currículo oficial del Curso de Orientación Universitaria de 1978 el estudio del origen de la vida en la materia de Geología, y de la evolución y la genética de poblaciones en la de Biología ${ }^{88}$.

Recibido: 21 de abril de 2010.

Aceptado: 21 de octubre de 2010.

87 Orden de 22 de marzo de 1975 (Boletín Oficial del Estado de 18 de abril, p. 8065).

88 Algunos de estos temas ya se encontraban en los textos de Biología del Curso de Orientación Universitaria de principios de los setenta de Salustio Alvarado y de Vicente Dualde (n. 1923). Aunque no se incluyeron en el programa oficial de 1975 (BOE de 6 de septiembre de 1975, p. 18926), sí fueron asumidos en 1978 (BOE de 17 de marzo de 1978, pp. 6445-6452). Alvarado, S. (1971), Biología, COU, Madrid, Agesa; Dualde, V. (1972), Biología, COU, Valencia, López Mezquita. 